\title{
CARACTERIZACIÓN FITOQUÍMICA, DETERMINACIÓN DEL CONTENIDO DE LIGNINA Y LA ACTIVIDAD ANTIOXIDANTE DE LOS CULMOS DE Guadua angustifolia KUNTH
}

Fecha de recepción: 12 de diciembre de 2014 •

Fecha de evaluación: 05 de mayo de 2015 • Fecha de aceptación: 23 de julio de 2015

\section{PHYTOCHEMICAL CHARACTERIZATION AND DETERMINATION OF ANTIOXIDANT ACTIVITY OF Guadua angustifolia Kunth CULMS}

Oscar Marino Mosquera ${ }^{1,3} \bullet$ Lina María González • Yeny Juliana Cortes • Juan Carlos Camargo²

RESUMEN

El objetivo del presente trabajo fue evaluar la presencia de metabolitos secundarios presentes en los culmos de Guadua angustifolia Kunth (guadua), así como determinar la actividad antioxidante de los mismos. Se caracterizaron fitoquímicamente por cromatografía de capa delgada (CCD) extractos de polaridades crecientes de culmos de guadua colectados en bosques naturales de cuatro sitios distintos del departamento de Risaralda (Colombia). En las muestras fueron detectados compuestos fitoquímicos correspondientes a alcaloides, fenoles, flavonoides, terpenos, saponinas triterpénicas y esteroidales. El contenido de lignina y la actividad antioxidante también fueron detectados por los métodos del $\mathrm{DPPH}^{*}$ y ABTS ${ }^{*}$. Se evaluó el contenido de fenoles y flavonoides totales, obteniéndose como resultado una actividad antioxidante y un contenido fenólico alto, en los extractos etanólicos. Adicionalmente, se determinó el perfil cromatográfico de los diferentes extractos mediante HPLC-DAD lo que permitió confirmar la presencia de compuestos fenólicos en los extractos de acetona y etanol.

Palabras claves: DPPH॰, ABTS ${ }^{*}$, HPLC-DAD.

1 Grupo de Biotecnología-Productos Naturales (GB-PN) Escuela de Tecnología Química, Universidad Tecnológica de Pereira (UTP), Colombia

2 Gestión en agrosistemas Trópicales andinos (GATA) Facultad de ciencias ambientales UTP

3 Correo-e: omosquera@utp.edu.co 


\section{ABSTRACT}

The aim of this study was to evaluate the presence of secondary metabolites in culms of Guadua angustifolia Kunth (guadua), so as to determine the antioxidant activity. Phytochemically were characterized by thin layer chromatography extracts whit polarities differents. Guadua culms were collected in natural stands located in four different sites of Risaralda state, (Colombia). Phytochemical compounds corresponding to alkaloids, phenols, flavonoids, terpenes, triterpene saponins and steroidal were detected. Besides, the methods of DPPH and ABTS were applied and lignin content and as well as the antioxidant capacity were also determined. Furthermore, the total content of phenols and flavonoids was assessed. An antioxidant activity and a high phenolic content within ethanol extracts were found as well. Additionally, the presence of phenolic compounds in acetone and ethanol extracts was confirmed by means of the chromatographic profile of the different extracts determined by HPLC-DAD.

Keywords: DPPH॰, ABTS*+, HPLC-DAD.

\section{INTRODUCCIÓN}

El uso de antioxidantes sintéticos es controversial debido a los múltiples reportes de su toxicidad. Por tal razón, la búsqueda de antioxidantes naturales de origen vegetal es de gran interés tanto para las industrias farmacéuticas como las de alimentos (1).

La actividad antioxidante de las plantas está asociada a compuestos fenólicos cuya estructura química con grupos hidroxilo, les permite actuar como antioxidantes primarios al inicio del proceso oxidativo o en la etapa de terminación, cuando se generan los radicales libres (2). La actividad antioxidante de los compuestos fenólicos protege al organismo contra las especies reactivas de oxígeno y los radicales libres implicados en la génesis del estrés oxidativo (3), el cual causa enfermedades crónicas y degenerativas, como el cáncer, la diabetes, las asociadas a problemas cardiovasculares y el Alzheimer (2).

Las especies de bambú pertenecen a la familia Poaceae, que crecen tanto en regiones templadas como en el trópico. Existen dos tribus de gran importancia económica y ecológica que son la Arundinarieae y la Bambuseae. La especie guadua (Guadua angustifolia Kunth) pertenece a la tribu Bambuseae, Subtribu Guadinae (4). En Colombia, los culmos de guadua han sido usados tradicionalmente en la construcción y en la elaboración de artesanías y son ampliamente utilizados por los productores rurales como una alternativa económica en la región cafetera (5). Recientemente, Camargo et al., (6) realizaron un estudio en el cual se pretendió conocer más a fondo las propiedades físico-mecánicas, el contenido de lignina y azúcares de esta especie con el fin de desarrollar técnicas y prácticas para un mejor conocimiento de la guadua y contribuir al desarrollo forestal del eje cafetero colombiano.

Diferentes especies de bambú han sido estudiadas por sus propiedades antioxidantes. Varios reportes indican que poseen esta actividad (7), (8), 
(9). Sin embargo, los trabajos basados en las actividades biológicas y fitoquímicas de guadua hasta el momento son inexplorados.

El objetivo de este estudio fue caracterizar los extractos de diferentes polaridades de los culmos de guadua por medio de CCD, cuantificar el contenido de lignina, fenoles y flavonoides totales y determinar su capacidad antioxidante por los métodos del $\mathrm{DPPH}^{\bullet}$ y $\mathrm{ABTS}^{\bullet}$.

\section{MATERIALES Y MÉTODOS}

\section{Reactivos, solventes y equipos}

Los solventes usados fueron de grado analítico y HPLC marca Mallinckrodt (Phillipsburg, NJ, USA), los reactivos de Trolox (ácido 6-hidroxi-2,5,7,8-tetrametilcromo-2-carboxílico), DPPH ${ }^{\bullet}$ (2,2-Difenil1-picrilhidrazilo), ABTS ${ }^{\bullet+}$ (ácido 2,2' azinobis-[3etilbenzotiazolina]-6-sulfonato de amonio), el reactivo de Folin \& Ciocalteu $2 \mathrm{~N}$ y el ácido gálico (ácido 3,4,5-trihidroxibenzoico) fueron marca SigmaAldrich (Saint Louis, MO, USA) y las cromatoplacas de Silica gel $F_{254} 0.2$ mm fueron Merck (Darmstadt, Germany). Para todas las determinaciones se utilizó un espectrofotómetro de microplacas Multiskan GO UV/Vis Thermo Scientific (Vantaa, Finlandia). Para la obtención de los perfiles cromatográficos se utilizó un HPLC Agilent 1100 (Los Ángeles, USA) dotado con un inyector manual Reodhyne, desgasificador, bomba cuaternaria, horno para la columna, detector de arreglo de diodos (DAD), columna Hypersil ODS $(5 \mu \mathrm{m}, 250 \times 4 \mathrm{~mm})$ y se utilizó el software de adquisición de datos ChemStation (Versión B.04.03).

\section{Preparación de la muestra}

El material vegetal de varios culmos de guadua se recolectó siguiendo la metodología propuesta por Camargo et al., (10), de los sitios: Finca el Bambusal (F.B), (Montenegro, Quindio; $4^{\circ}$ 51.8' $\mathrm{N}, 75^{\circ} 79.0^{\prime} \mathrm{W}$ ) a $1200 \mathrm{msnm}$, Jardín Botánico de la
Universidad Tecnológica de Pereira (J.B-UTP) a 1450 msnm, (Pereira, Risaralda; $4^{\circ} 47^{\prime} \mathrm{N}, 75^{\circ} 41^{\prime} \mathrm{W}$ ), Finca Nápoles (F.N) a 1150 msnm, (Montenegro, Quindío; $4^{\circ} 55.5^{\prime} \mathrm{N}, 75^{\circ} 80.2^{\prime} \mathrm{W}$ ) y Finca Yarima (F.Y) (Pereira, Risaralda; $4^{\circ} 47^{\prime} 30^{\prime \prime} \mathrm{N}$; $4^{\circ} 48^{\prime} 20^{\prime \prime} \mathrm{N} ; 75^{\circ} 45^{\prime} 50^{\prime \prime} \mathrm{W}$; $\left.75^{\circ} 46^{\prime} 40^{\prime \prime} \mathrm{W}^{\prime}\right)$ a $1150 \mathrm{msnm}$. En total se analizaron 24 muestras de culmos (seis por cada sitio.

Se utilizó la metodología descrita por Mosquera et al., (11). Cinco gramos de material vegetal seco y molido de cada una de las 24 muestras colectadas de los culmos de guadua, se sometieron a extracción inicialmente con acetona a $45^{\circ} \mathrm{C}$ por 6 horas (dos veces). Posteriormente, se extrajeron sucesivamente con agua a $80^{\circ} \mathrm{C}$ por 1 hora (dos veces) y finalmente con etanol a $60^{\circ} \mathrm{C}$ por 6 horas (dos veces) para obtener los respectivos componentes presentes en las diferentes muestras.

\section{Determinación de fitocompuestos por cromatografía de capa delgada (CCD)}

Los extractos de acetona y etanol de guadua de todos los sitios fueron caracterizados por CCD siguiendo el protocolo de Wagner and Bladt., (12). Se emplearon cromatoplacas de sílica gel $60 \mathrm{~F}_{254}(5 \times 4$ $\mathrm{cm})$, los extractos se sembraron 30 veces a una concentración de $1000 \mathrm{mg} / \mathrm{L}$, las placas se realizaron por triplicado. Los extractos de acetona fueron eluidos con el sistema n-hexano-acetato de etilo (AcOEt) (85:15) y los extractos de etanol con AcOEt-MeOH$\mathrm{H}_{2} \mathrm{O}$ (80:15:5); los núcleos fitoquímicos fueron analizados con los reveladores de Dragendorff, $\mathrm{FeCl}_{3}$ al $5 \%$ en $\mathrm{HCl} 0.5 \mathrm{~N}, \mathrm{AlCl}_{3}$ al $1 \%$ en $\mathrm{EtOH}$, LiebermannBurchard, Ácido fosfomolíbdico al 5\% en EtOH, $\mathrm{KOH}$ en $\mathrm{EtOH}$ al 5\% para detectar alcaloides, fenoles, flavonoides, saponinas, terpenos y antraquinonas, respectivamente. Se obtuvo un registro fotográfico de cada una de las placas y posteriormente cada fotografía fue analizada con el software ImageJ para cuantificar la intensidad de cada una de las manchas correspondientes a cada núcleo fitoquímico. 
Extracción y cuantificación de lignina ácida insoluble

Para la extracción de la lignina insoluble se siguió la metodología descrita por Mosquera et al (13). El porcentaje de lignina insoluble en la muestra se determinó, mediante la siguiente ecuación donde \% lignina insoluble $=($ peso del residuo $/ 250 \mathrm{mg}) \times 100$.

\section{Determinación de la capacidad antioxidante} por el método del DPPH

La actividad antioxidante se midió a través del método del radical $\mathrm{DPPH}{ }^{*}$ descrito por Brand-Williams et al., (14) con modificaciones. La capacidad captadora de electrones se determinó mediante la reacción de $100 \mu \mathrm{L}$ de una solución de DPPH• a $20 \mathrm{mg} / \mathrm{L}$ y $25 \mu \mathrm{L}$ de una solución del extracto a $1000 \mathrm{mg} / \mathrm{L}$ durante media hora en condiciones de oscuridad y a temperatura ambiente $\left(A_{\text {muestra }}\right)$. Como control negativo, se utilizó el solvente en el cual habían sido solubilizados los extractos $\left(A_{\text {control (- }- \text { ) }}\right.$ y como control positivo hidroquinona a $1000 \mathrm{mg} / \mathrm{L}$. La reducción del radical fue medida a $\lambda$ $=517 \mathrm{~nm}$. El porcentaje de decoloración del DPPH ${ }^{\circ}$, se determinó mediante la siguiente ecuación:

$$
\begin{gathered}
\text { \% Actividad antioxidante }=\left[\left(\mathrm{A}_{\text {Control (-) }}-\mathrm{A}_{\text {muestra }} /\right.\right. \\
\left.\mathrm{A}_{\text {Control (-) })}\right] \times 100
\end{gathered}
$$

Se realizó una curva de calibración de Trolox a las concentraciones de 1, 2, 4, 8, 16, 32 y $64 \mu \mathrm{M}$ para expresar la capacidad antioxidante en equivalentes Trolox ( $\mu$ mol Trolox/g extracto).

\section{Determinación de la capacidad antioxidante por el método del ABTS ${ }^{+}$}

La capacidad de inhibir el radical 2,2'-azino-bis (3-etilbenzotiazolina)-6-sulfonato de amonio (ABTS ${ }^{\bullet}$ ) de los extractos estudiados se determinó siguiendo el método propuesto por Re et al., (15) con algunas modificaciones. El radical se generó mediante la oxidación del ABTS a $3.5 \mathrm{mM}$ con persulfato de potasio a $1.25 \mathrm{mM}$. Para la realización del ensayo, se tomaron
$6 \mu \mathrm{L}$ de extracto a $1000 \mathrm{mg} / \mathrm{L}$ y se mezclaron con 294 $\mu \mathrm{L}$ de la solución de $\mathrm{ABTS}{ }^{\bullet+}$ previamente preparada y ajustada a un valor de absorbancia de $0.700 \pm 0.002$ con etanol. Esta mezcla se dejó reaccionar durante media hora en ausencia de luz y a temperatura ambiente. La reducción del ABTS ${ }^{*+}$ se medió a $\lambda=732$ $\mathrm{nm}$. Los controles negativo y positivo fueron los mismos utilizados en el ensayo con DPPH ${ }^{*}$ al igual que la ecuación empleada para la determinación de la actividad antioxidante. Se realizó una curva de calibración de Trolox a las concentraciones de 1, 5, 10, 20, 40, 80 y $100 \mu \mathrm{M}$ para expresar la capacidad antioxidante en equivalentes Trolox ( $\mu \mathrm{mol}$ Trolox/g extracto).

\section{Determinación del contenido de fenoles totales}

Para la cuantificación del contenido fenólico se siguió la metodología descrita por Magalhães et al., (16), en la cual a $50 \mu \mathrm{L}$ del extracto a $100 \mathrm{mg} / \mathrm{L}$ se le adicionaron $50 \mu \mathrm{L}$ del reactivo de Folin-Ciocalteu $(1: 50, v / v)$ e inmediatamente después se adicionaron $100 \mu \mathrm{L}$ de una solución de $\mathrm{NaOH}(0.35 \mathrm{M})$. Esta mezcla se dejó reaccionar por tres minutos y se midió la absorbancia a $760 \mathrm{~nm}$. Para esta determinación se realizó una curva de calibración de ácido gálico a 0 , 2, 4, 8 y $16 \mu \mathrm{g}$ Ácido gálico/mL.

\section{Determinación del contenido de flavonoides totales}

Para la determinación del contenido de flavonoides totales, se siguió la metodología descrita por Kim et al., (17) con modificaciones. Se preparó la mezcla constituida por $20 \mu \mathrm{L}$ del extracto a $50 \mathrm{mg} / \mathrm{L}$ más 115 $\mu \mathrm{L}$ de $\mathrm{H}_{2} \mathrm{O}$ y se le adicionó $7.5 \mu \mathrm{L}$ de $\mathrm{NaNO}_{2}(5 \%)$. Se dejó reaccionar durante 5 minutos y posteriormente se adicionaron $30 \mu \mathrm{L}$ de $\mathrm{AlCl}_{3}(2.5 \%)$, permitiendo esta reacción por 6 minutos. Luego de este periodo se agregaron $50 \mu \mathrm{L}$ de $\mathrm{NaOH}(1 \mathrm{M})$ e inmediatamente $50 \mu \mathrm{L}$ de $\mathrm{H}_{2} \mathrm{O}$; después de 5 minutos, se midió la absorbancia a $500 \mathrm{~nm}$. Para la cuantificación de flavonoides totales se realizó una curva de calibración de 0, 0.05, 0.1, 0.2, 0.4 y $0.8 \mu \mathrm{g} \mathrm{Kaempferol} / \mathrm{mL}$. 


\section{Evaluación del perfil cromatográfico de compuestos fenólicos}

Se utilizó un equipo HPLC Agilent 1100, las condiciones cromatográficas fueron muy similares a las empleadas por Turumtay et al., (18). Para esta determinación se utilizó una Columna Hypersil ODS (5 $\mu \mathrm{m}, 250 \times 4 \mathrm{~mm}$ ), la fase móvil fue ácido fórmico al $1 \%(A)$ y metanol $(B)$ con el siguiente gradiente: $0-3$ $\min 5 \% \mathrm{~B}$; 3-8 $\min 5-15 \% \mathrm{~B}$; 8-10 $\min 15-20 \% \mathrm{~B} ; 10-12$ $\min 20-25 \%$ B; $12-20$ min 25-40\% B; $20-30 \min 40-80 \%$ B. La temperatura de la columna fue de $25^{\circ} \mathrm{C}$ con un flujo de $1 \mathrm{~mL} / \mathrm{min}$, se utilizó un detector de arreglo de diodos (DAD) a 280 y $315 \mathrm{~nm}$. Se inyectaron $20 \mu \mathrm{L}$ de cada muestra, a una concentración de 1000 mg/L.

\section{Análisis estadístico}

Inicialmente, se realizaron estadísticas descriptivas teniendo en cuenta estadísticos que indicaban la dispersión de los datos como la desviación estándar (DS). Estas se realizaron para cada una de las tres mediciones de manera independiente. El análisis estadístico se realizó en el software GraphPad Prism versión 5. También se realizaron correlaciones entre las variables analizadas, teniendo en cuenta el coeficiente de correlación de Pearson (R) y la probalidad $(p<0.05)$ para detectar relaciones entre las mismas. En el análisis de los cromatoplacas se utilizó el software ImageJ versión 1.48 para cuantificar los metabolitos secundarios presentes en los extractos de culmos de guadua.

\section{RESULTADOS Y DISCUSIÓN}

\section{Caracterización fitoquímica por cromatografia}

de capa delgada (CCD) de los extractos

de G. angustifolia

En la figura 1 se presenta una cromatoplaca de la caracterización por CCD de fenoles en los extractos de acetona de culmos de guadua de los sitios de estudio. Es posible observar manchas azules que indican la presencia de estos metabolitos secundarios. De manera similar, se realizó la caracterización de cada uno de los núcleos químicos evaluados por CCD.

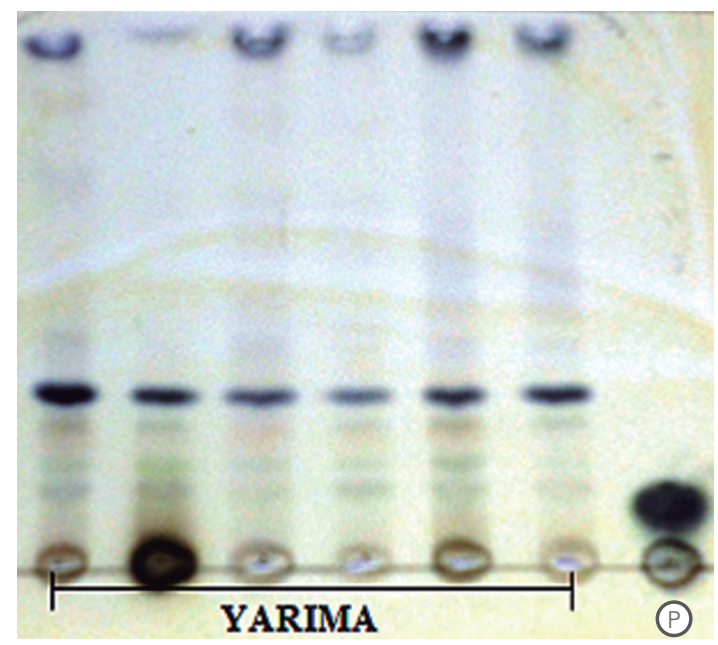

Figura 1. Placa de CCD de los extractos de acetona de la finca Yarima. (P) Control positivo (Catecol). Cromatoplacas de Sílica gel $60 \mathrm{~F}_{25}$ $(5 \times 4 \mathrm{~cm})$; fase móvil, $n$-hexano-EtOAc (85:15). Revelador: $\mathrm{FeCl}_{3}$ al $5 \%$ en $\mathrm{HCl} 0.5 \mathrm{~N}$

En las muestras de culmos de guadua de todos los sitios, se encontraron alcaloides, fenoles, flavonoides, saponinas y terpenos, tanto en los extractos de acetona como en los de etanol (Figura 2). Los núcleos fenólicos, flavonoides y saponinas, que fueron más abundantes lo cual concuerda con lo reportado por Zhang et al., (19), (20) quienes estudiaron la presencia de compuestos triterpénicos y flavonoides como la orientina, homoorientina, vitexina e isovitexina en la especie de bambú Phyllostachys nigra. En ninguna de las zonas se detectó la presencia de antraquinonas.

Muestras de culmos colectados en la F.N, mostraron altos contenidos de saponinas en ambos extractos. Las saponinas son compuestos que las plantas producen como mecanismo de defensa contra patógenos y herbívoros (21). Altos niveles de estos 

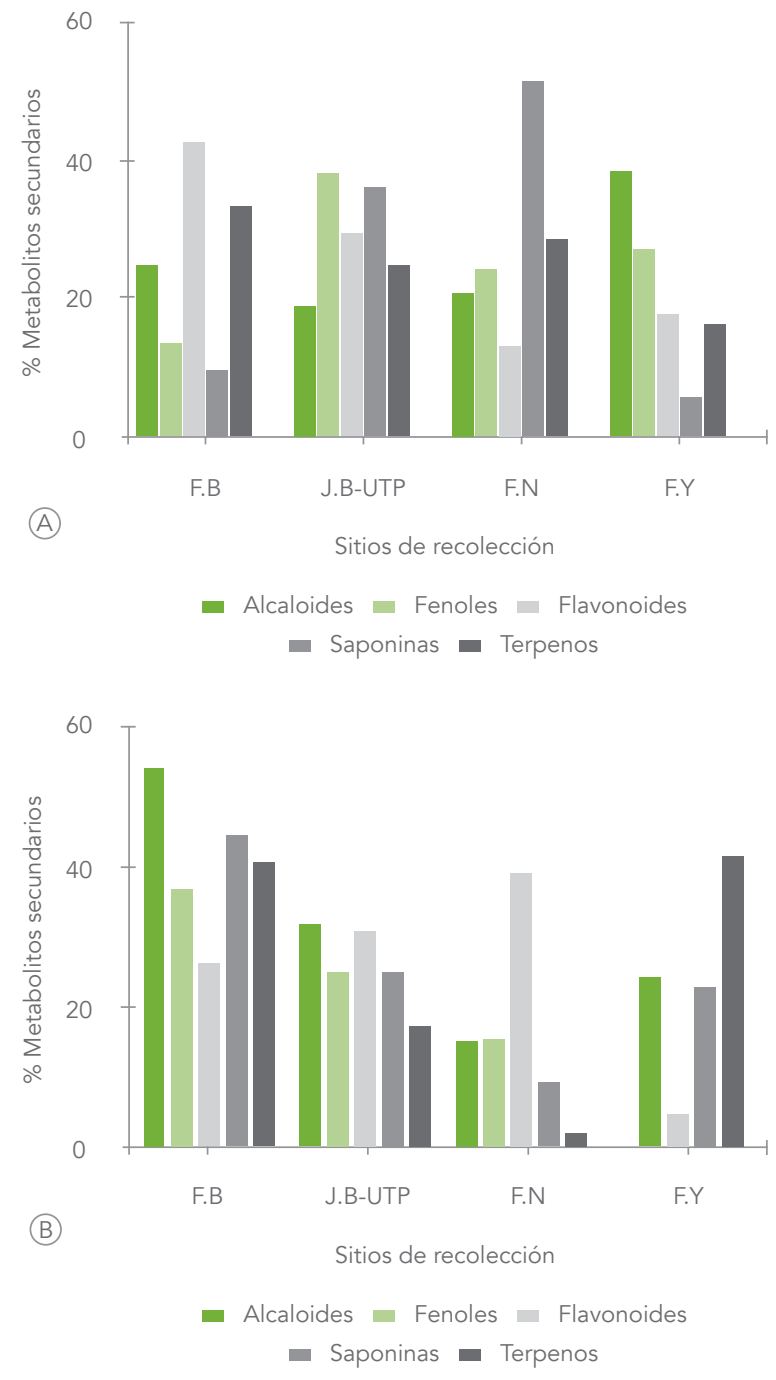

Figura 2. Porcentaje de metabolitos secundarios determinados por CCD en los extractos de (A) acetona, (B) etanol de culmos de Guadua angustifolia Kunth. La abundancia relativa de cada mancha fue determinada usando el software Image J. Finca el Bambusal (F.B.), Jardín Botánico-UTP (J.B - U.T.P), Finca Nápoles (F.N) y Finca Yarima (F.Y).

metabolitos, pueden estar influenciados por factores abióticos y bióticos como la temperatura, humedad, luz, fertilidad del suelo, competencia con cultivos o plantas vecinos e interacciones con organismos patógenos como bacterias, hongos, virus, nematodos e insectos (22). Esta condición podría estar asociada a un posible ataque de algún taladrador $\mathrm{u}$ otro enemigo natural, de tal manera que como estrategia su biogénesis incrementó la producción de saponinas con el fin de superar esta amenaza.

Los extractos de acetona, presentaron un alto contenido de metabolitos secundarios en comparación con los extractos de etanol, debido a que este fue el primer disolvente de extracción., En este sentido, de la F.B se obtuvieron los culmos con mayor contenido de dichos compuestos. Por esta razón, podría inferirse que algunas condiciones de sitio están incidiendo en la biogénesis de metabolitos secundarios en guadua.

Extracción y cuantificación de lignina ácida insoluble

En el porcentaje de lignina ácida insoluble en los culmos de guadua no se observaron diferencias significativas ( $p>0.05$ ) entre los sitios (Figura 3). Estos resultados son similares con lo reportado por Sun et al., (8) para la especie Phyllostachys pubescen y por Mosquera et al., (11) para esta misma especie. Se demuestra así, la biodisponibilidad de lignina en culmos procedentes de todos los sitios incluidos. Teniendo en cuenta que la lignina tiene usos potenciales en la industrias química y de polímeros (23), podría ser la guadua una especie de interés para futuras investigaciones.

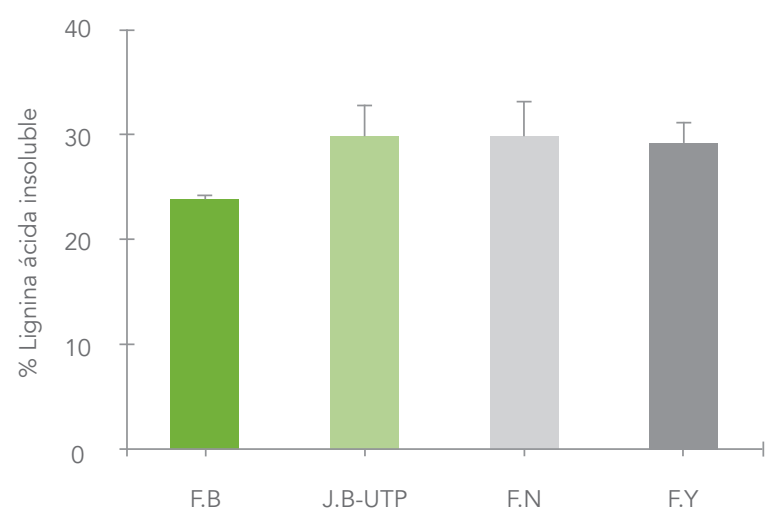

Figura 3. Porcentaje de lignina ácida insoluble en las muestras de culmos de Guadua angustifolia de diferentes sitios. Líneas sobre la barra son la desviación estándar. 


\section{Capacidad antioxidante} Método del DPPH ${ }^{\circ}$

En la evaluación de la actividad antioxidante mediante el método DPPH, los extractos de acetona de los sitios de estudio, presentaron una actividad menor a $46 \mu \mathrm{mol}$ trolox/g extracto como se observa en la figura 4(A).

De otro lado, todos los extractos etanólicos (Figura $4(B))$ presentaron actividad antioxidante mayor a $80 \mu \mathrm{mol}$ trolox/g extracto. Para los extractos de agua la actividad antioxidante fue mayor a $50 \mu \mathrm{mol}$ trolox/g extracto para los sitios F.B, J.B-UTP y F.N, mientras que en la F.Y, la actividad antioxidante fue menor a $50 \mu \mathrm{mol}$ trolox/g extracto (Figura $4(\mathrm{C})$ ). No obstante, las diferencias no fueron estadísticamente significativas ( $p>0.05)$ entre sitios.

Estos resultados se relacionan con la caracterización por CCD de los fenoles y flavonoides en los extractos de acetona y etanol. Por tal razón, la actividad antioxidante podría ser atribuida a dichos núcleos. Los datos anteriores indican que el mejor solvente de extracción para compuestos con actividad antioxidante fue el etanol, lo cual concuerda con estudios realizados por Shang et al.,(9) quienes reportaron actividad antioxidante por el método del $\mathrm{DPPH}^{\bullet}$ en los extractos hidroetanólicos de la especie de bambú Phyllostachys nigra.

\section{Método del ABTS ${ }^{\circ+}$}

El valor de la actividad antioxidante por ABTS ${ }^{\bullet+}$ para los extractos de acetona y agua del sitio el Bambusal, presento un valor superior a $100 \mu \mathrm{mol}$ trolox/g extracto y significativamente diferente $(p<0.05)$ respecto a los demás sitios evaluados (Figura 5 (A y C)). Los extractos de etanol presentaron un comportamiento similar a los obtenidos por el método del $\mathrm{DPPH}^{\bullet}$, dado que presentaron una actividad antioxidante alta. Así, se confirma que el mejor solvente para la extracción de metabolitos con actividad antioxidante fue etanol (Figura 5(B)).
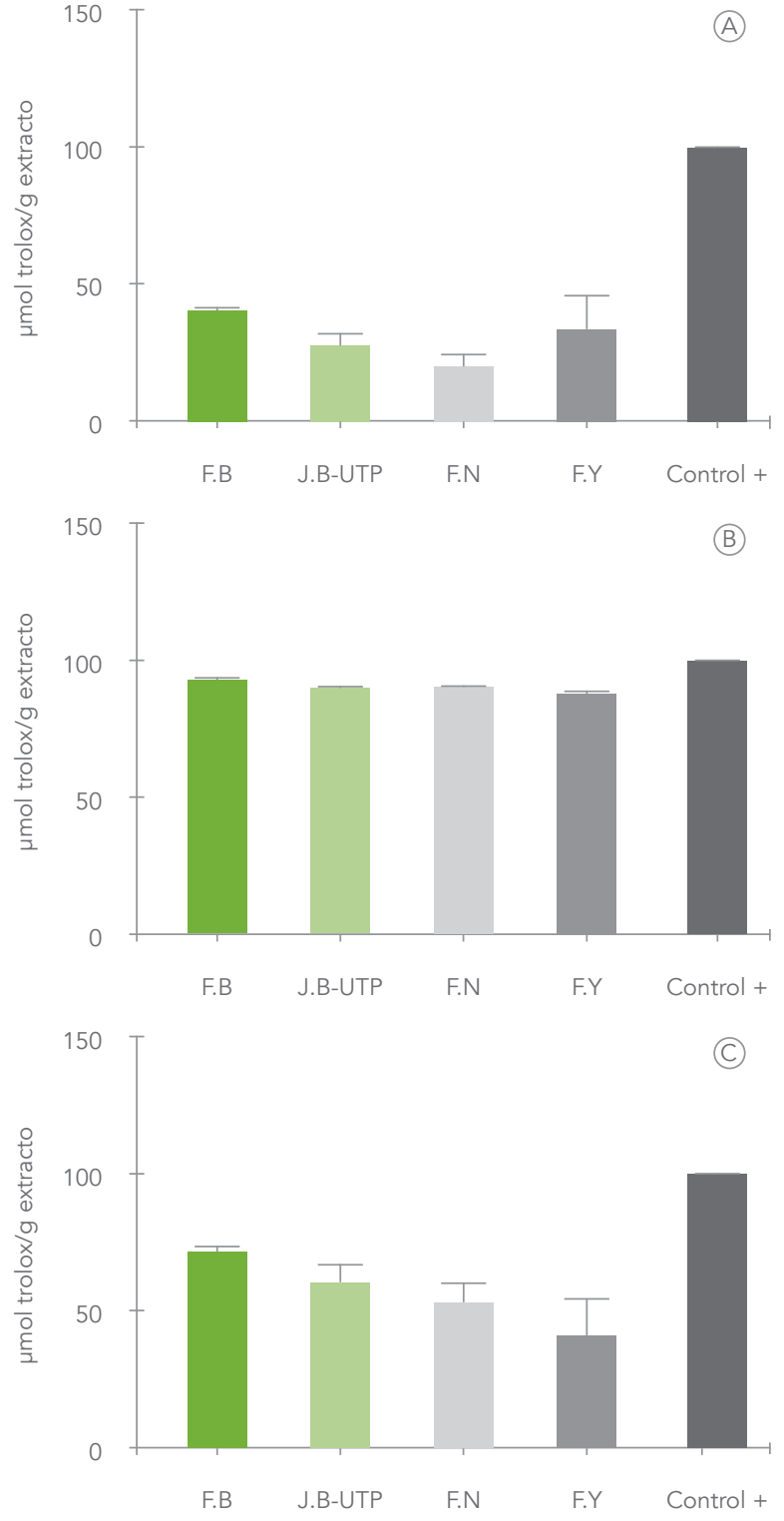

Figura 4. Porcentaje de la actividad antioxidante por el método del $\mathrm{DPPH}^{\bullet}$ en los extractos de (A) acetona, (B) etanol, (C) agua de muestras de culmos de Guadua angustifolia. Control positivo (Hidroquinona, 1000 $\mathrm{mg} / \mathrm{L}$ ). Líneas verticales sobre sobre las barras son la desviación estándar.

De otro lado, una correlación significativa $(p<0.05)$ fue encontrada entre los ensayos del DPPH ${ }^{\bullet}$ y ABTS ${ }^{\bullet+}$ $(R=0.676, R=0,459$ y $R=0,748$ para los extractos de acetona, etanol y agua respectivamente (Tabla 1). 
Tabla 1. Coeficientes de correlación de Pearson (R) entre los métodos

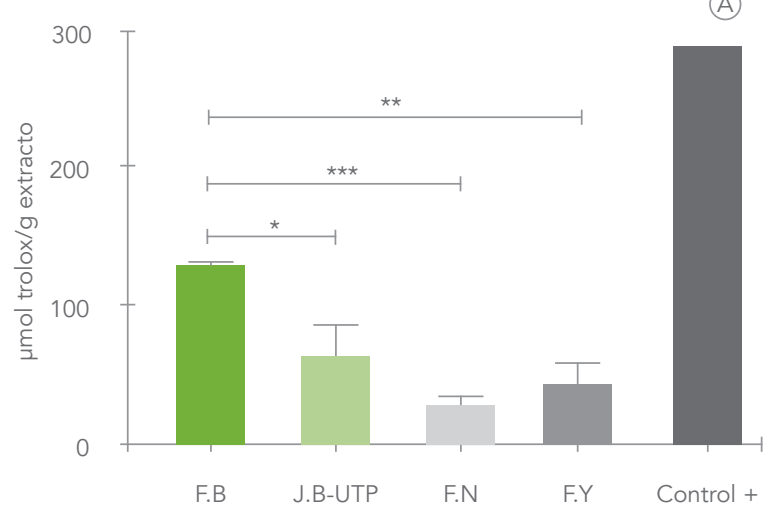

(B)
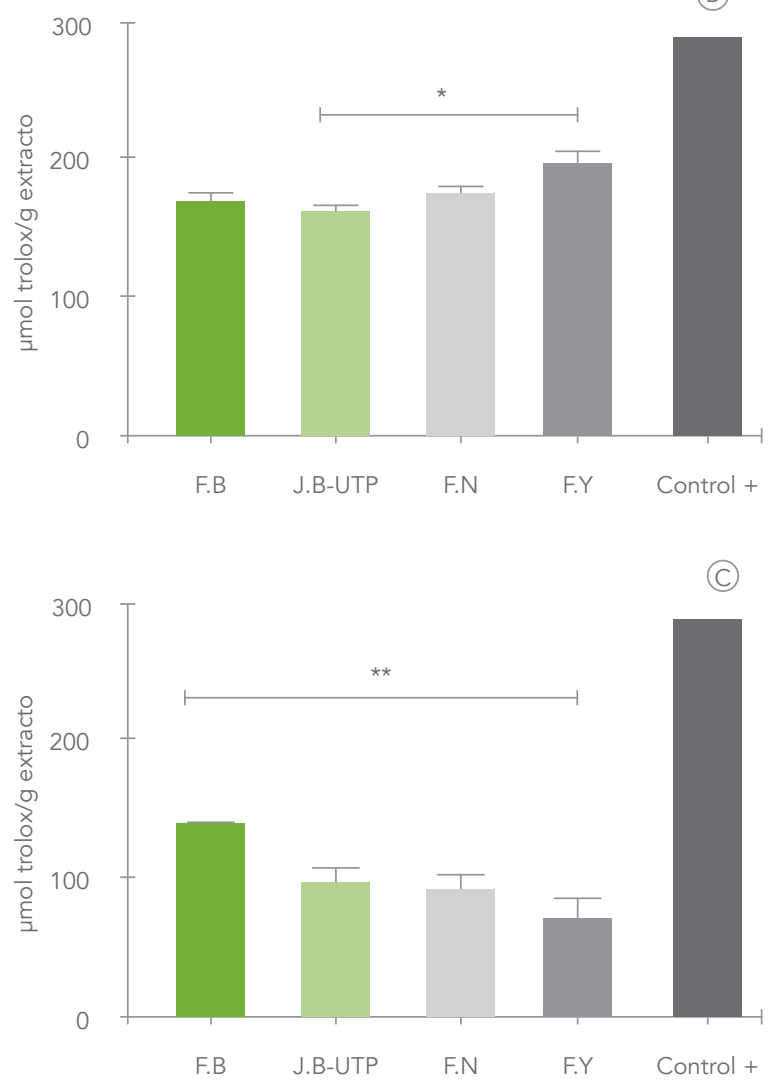

Figura 5. Porcentaje de la actividad antioxidante por el método del $\mathrm{ABTS}^{*+}$ en los extractos de (A) acetona, (B) etanol, (C) agua de muestras de culmos de Guadua angustifolia. Control positivo (Hidroquinona, 1000 $\mathrm{mg} / \mathrm{L}$ ). Líneas verticales sobre las barras son la desviación estándar. Líneas horizontales sobre las barras son diferencias estadísticamente significativas

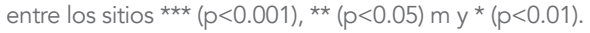

del DPPH ${ }^{\bullet}$ ABTS $^{\bullet+}$ para los extractos de acetona, etanol y agua.

\begin{tabular}{cc}
\hline Extracto & $\mathbf{R}$ \\
\hline Acetona & $0.676^{\star}$ \\
\hline Etanol & $0.4589^{\star}$ \\
\hline Agua & $0.7481^{\star}$ \\
\hline
\end{tabular}

* Significativo con $\mathrm{P}<0.05$

Relaciones entre la actividad antioxidante y el contenido de fenoles y flavonoides totales

Entre los extractos de acetona y etanol se pudo observar que el contenido de compuestos fenólicos fue mayor que en los acuosos (figuras 6A, 6B y 6C). Así mismo, los resultados de correlación obtenidos por el método del DPPH se presentan en la tabla 2.

Tabla 2. Coeficientes de correlación entre el contenido de fenoles y flavonoides con DPPH• para los extractos de acetona, etanol y agua.

\begin{tabular}{ccc}
\hline Extracto & Fenoles & Flavonoides \\
\hline Acetona & $0.4330^{\star}$ & $0.3133^{\text {ns }}$ \\
\hline Etanol & $0.4062^{\text {ns }}$ & $0.2372^{\text {ns }}$ \\
\hline Agua & $0.3804^{\text {ns }}$ & $0.0649^{\text {ns }}$ \\
\hline
\end{tabular}

ns = no significativo

* Significativo con $\mathrm{P}<0.05$

Kim et al., (24) encontraron unas correlaciones bajas $(R=0.2565)$ entre el contenido de fenoles totales y la actividad antioxidante empleando el método del $\mathrm{DPPH}^{\bullet}$ en diferentes extractos acuosos. En este trabajo, se encontraron diferencias significativas $(p<0.05)$ entre F.B y los demás sitios para los extractos de acetona (Figura $5(\mathrm{~A})$ ); mientras que para los extractos etanólicos no se presentaron diferencias significativas $(P>0.05)$ en el contenido de fenoles (Figura 6 (B)). Los extractos acuosos presentaron un contenido bajo de fenoles con respecto a los 
extractos de acetona y etanol (Figura 6 (C)). Además, se presentaron diferencias significativas $(p<0.05)$ entre muestras de los sitios F.B y F.Y.

Por otra parte, la correlación entre el contenido de flavonoides y el método del DPPH', también presentó coeficientes de correlación bajos. Estos resultados demuestran que la actividad antioxidante de los extractos de acetona podría atribuirse a los compuestos fenólicos y la actividad antioxidante de los extractos de etanol podría atribuirse a otro tipo de compuestos, dado que en la marcha fitoquímica se encontró diversidad de metabolitos secundarios.

El contenido de flavonoides en los extractos de acetona y etanol fue mayor que en los acuosos (figura 7), presentando de esta manera un comportamiento similar a los resultados obtenidos en el contenido de fenoles. Además, se encontraron diferencias significativas $(p<0.05)$ entre las muestras de F.B y los demás sitios estudiados para los extractos de acetona (Figura 7 (A)), mientras que para los extractos etánolicos y acuosos, no hubo diferencias significativas $(p>0-05)$ en el contenido de flavonoides (Figura $7(B, C)$ ).

\section{Evaluación del perfil cromatográfico}

Los extractos de acetona, etanol y agua de los culmos de guadua presentaron absorciones en el UV-visible en el rango de 280-315 nm Esto confirma la presencia de compuestos fenólicos en dichos extractos. Todas las muestras de las zonas estudiadas, presentaron señales características con tiempos de retención $\left(t_{R}\right)$ iguales como se presenta en la tabla 3 con sus correspondientes picos de absorción.

El perfil cromatográfico del extracto de acetona (B1-3) se presenta en la figura 8 , el cual presenta bandas de absorción fuertes a 235, 315 y un hombro a $300 \mathrm{~nm}$ para un $t_{R}$ de 17.413 min. Este extracto tuvo un porcentaje de actividad antioxidante de $91.02 \%$ y $66.73 \%$ a través de los ensayos DPPH• y ABTS ${ }^{\bullet}$, respectivamente. Lo cual podría corresponder al ácido caféico y sus derivados (25). También se evidenció la presencia
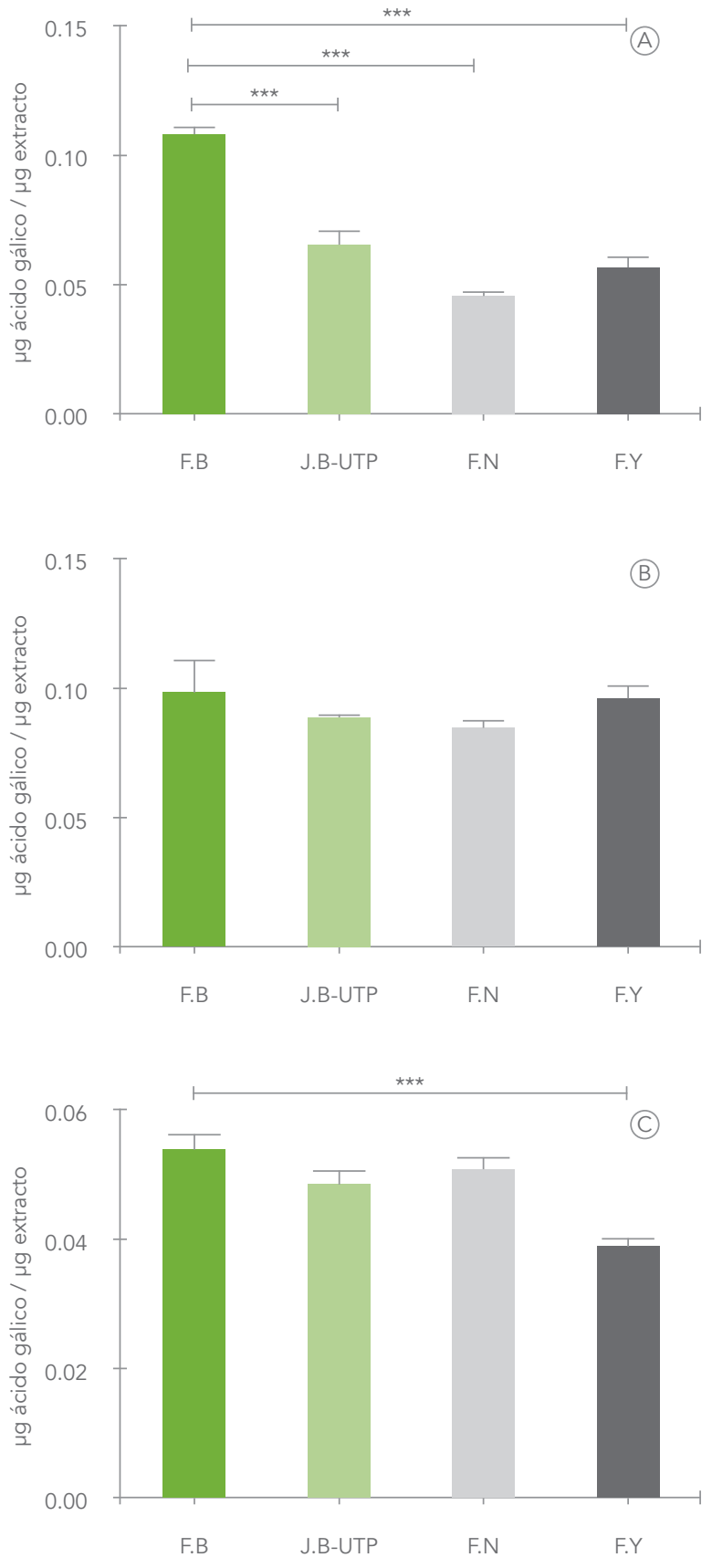

Figura 6. Contenido de fenoles totales en los extractos de (A) acetona, (B) etanol, (C) agua de muestras de culmos de Guadua angustifolia. Líneas verticales sobre las barras son la desviación estándar. Líneas horizontales sobre las barras son diferencias estadísticamente significativas entre los sitios *** $(p<0.001)$, ${ }^{\star *}(p<0.05) m$ y $(p<0.01)$. 

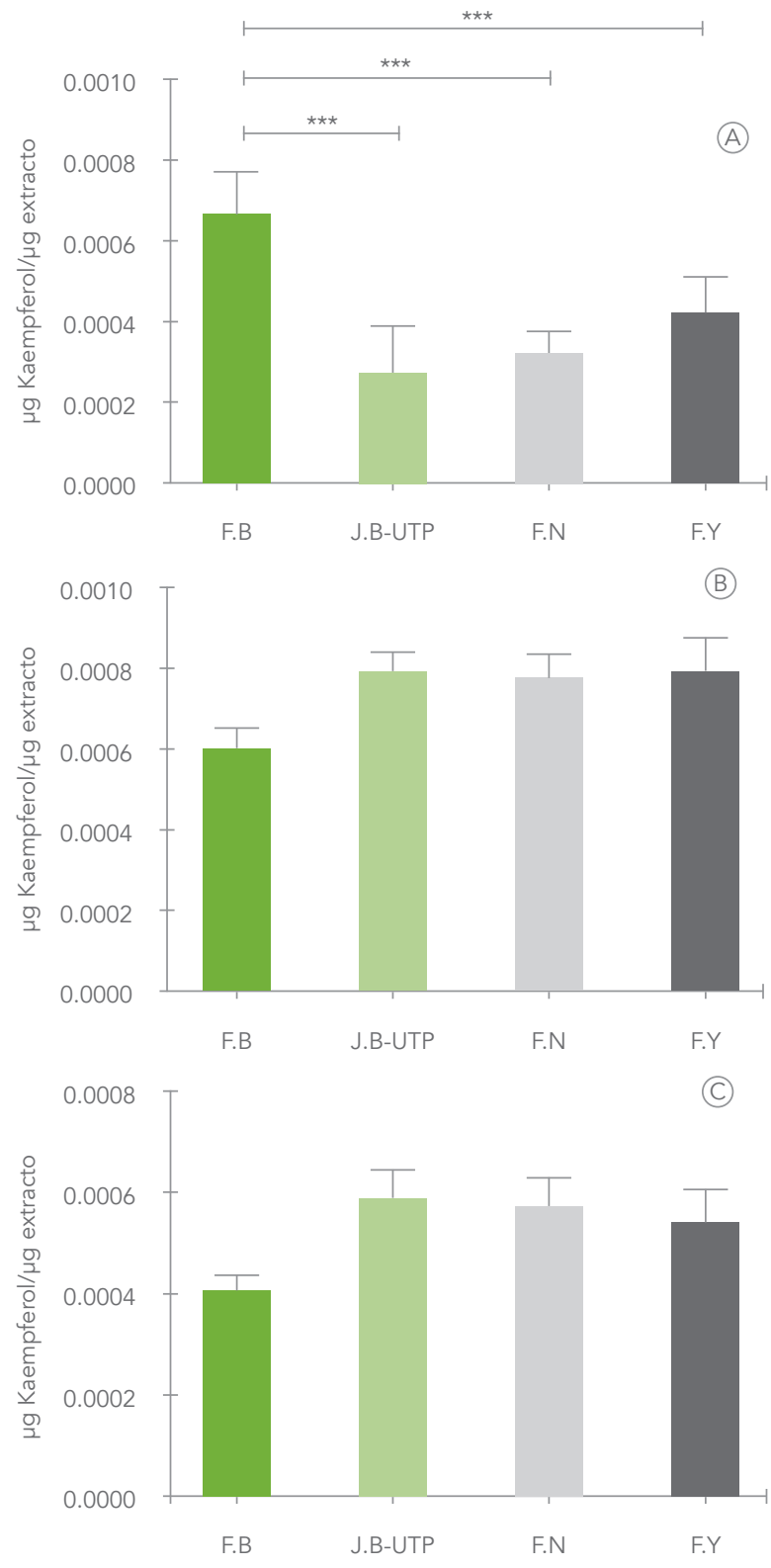

Figura 7. Contenido de Flavonoides en los extractos de (A) acetona, (B) etanol, (C) agua de muestras de culmos de Guadua angustifolia. Líneas verticales sobre las barras son la desviación estándar. Líneas horizontales sobre las barras son diferencias estadísticamente significativas entre los sitios ${ }^{\star \star *}(p<0.001),{ }^{\star *}(p<0.05) m y^{*}(p<0.01)$.

de fenoles simples con bandas de absorción a $270 \mathrm{~nm}$ y de lignanos con bandas de absorción simultaneas a 230 y $280 \mathrm{~nm}$. Así, se confirma que los extractos de los culmos de guadua contienen compuestos fenólicos con actividad antioxidante. De esta manera, esta condición resalta la potencialidad de la especie para la obtención de compuestos antioxidantes que podrían ser empleados en la industria alimenticia y cosmética, además de los atributos que hoy en día permiten usarla para aplicaciones en construcción.

\section{CONCLUSIONES}

Los extractos de acetona y etanol de culmos de guadua colectados en los sitios estudiados contienen los núcleos químicos de alcaloides, fenoles, flavonoides, terpenos, saponinas triterpénicas y esteroidales, con mayor abundancia de saponinas en los extractos de acetona; así, como fenoles y flavonoides en los de etanol.

La mayor actividad antioxidante encontrada en los extractos de etanol por el método del $\mathrm{DPPH}^{\bullet} \mathrm{y}$ $\mathrm{ABTS}^{\bullet+}$, indican que el etanol es el mejor solvente de extracción de este tipo de metabolitos en la especie de bambú guadua. De igual manera, el mayor contenido de fenoles y flavonoides en los extractos de acetona y etanol, sugieren que la actividad antioxidante podría ser atribuida a este tipo de compuestos, lo cual fue confirmado en la evaluación del perfil cromatográfico en donde se presentaron picos de absorción característicos de compuestos fenólicos.

Tabla 3. Tiempos de retención ( $t_{\mathrm{p}}$ ) obtenidos a través del perfil cromatográfico del extracto de acetona de culmos de Guadua angustifolia y las longitudes de onda para sus respectivos espectros UV.

\begin{tabular}{c|c|c} 
Señal $\mathbf{N}^{\circ}$ & $\mathbf{t}_{\mathbf{R}}(\mathbf{m i n})$ & $\boldsymbol{\lambda}_{\max }(\mathbf{n m})$ \\
\hline $\mathbf{1}$ & 13.876 & 225,260 \\
\hline $\mathbf{2}$ & 15.282 & $225,260,295$ \\
\hline $\mathbf{3}$ & 15.789 & 230,280 \\
\hline $\mathbf{4}$ & 17.413 & $235,300 \mathrm{~h}, 315$ \\
\hline $\mathbf{5}$ & 21.060 & $250,290 \mathrm{~h}, 320$
\end{tabular}

h=hombro 
(A)

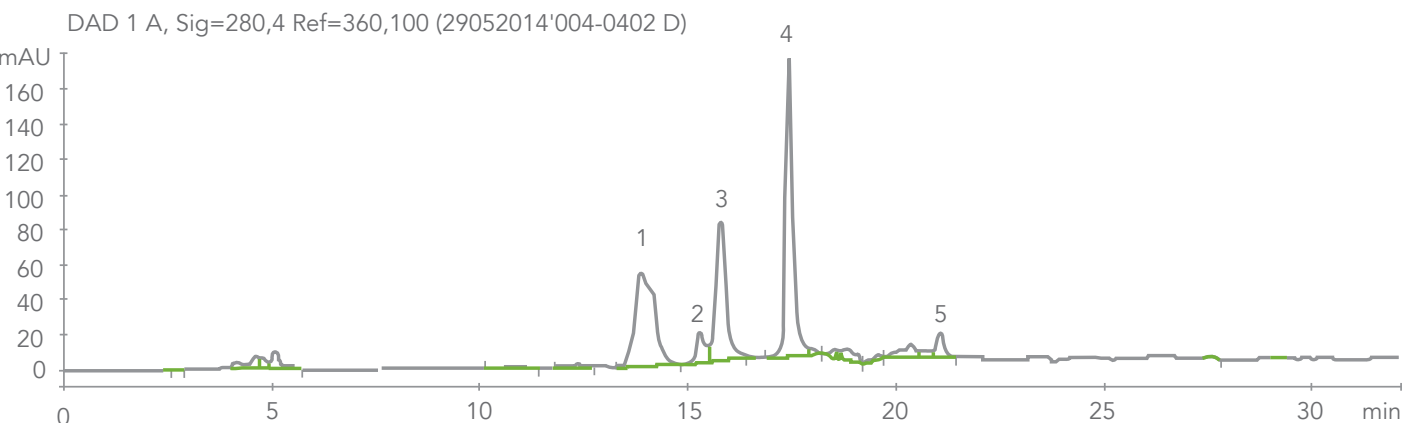

(B)

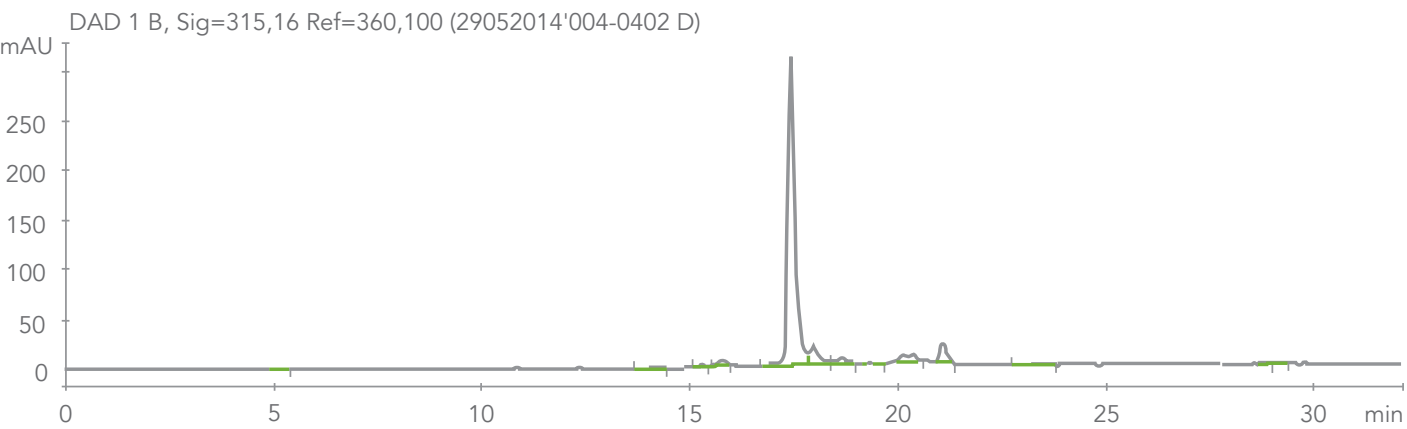

Figura 8. Perfil cromatográfico del extracto de acetona de culmos de Guadua angustifolia a longitud de onda 280 y $315 \mathrm{~nm}$. Sistema de elución gradiente $0-100 \%$ A (solvente A metanol, solvente B ácido fórmico al 1\%).

\section{REFERENCIAS}

1. Jun H-I, Kim B-T, Song G-S, Kim Y-S. Structural characterization of phenolic antioxidants from purple perilla (Perilla frutescens var. acuta) leaves. Food Chemistry. 2014;148:367-72.

2. Chen Z, Bertin R, Froldi G. EC50 estimation of antioxidant activity in DPPH assay using several statistical programs. Food Chemistry. 2013;138(1):414-20.

3. Siger A, Czubinski J, Kachlicki P, Dwiecki K, Lampart-Szczapa E, Nogala-Kalucka M. Antioxidant activity and phenolic content in three lupin species. Journal of Food Composition and Analysis. 2012;25(2):190-7.

4. Kelchner SA. Higher level phylogenetic relationships within the bamboos (Poaceae: Bambusoideae) based on five plastid markers. Molecular Phylogenetics and Evolution. 2013;67(2):404-13.
5. Kleinn C, Morales-Hidalgo D. An inventory of Guadua (Guadua angustifolia) bamboo in the Coffee Region of Colombia. European Journal of Forest Research. 2006;125(4):361-8.

6. Camargo JC, Rodriguez JA, Niño J, Mosquera OM, Ríos AM, Cortes YJ, et al. Desarrollo tecnológico para optimizar la calidad de los productos obtenidos de bosques de Guadua: Definiendo la madurez de los culmos y mejorando los procesos de organización. Pereira, Risaralda 2011.

7. Li M-F, Sun S-N, Xu F, Sun R-C. Microwave-assisted organic acid extraction of lignin from bamboo: Structure and antioxidant activity investigation. Food Chemistry. 2012;134(3):1392-8.

8. Sun S-L, Wen J-L, Ma M-G, Sun R-C, Jones GL. Structural features and antioxidant activities of degraded lignins from steam exploded 
bamboo stem. Industrial Crops and Products. 2014;56:128-36.

9. Shang YF, Kim SM, Um B-H. Optimisation of pressurised liquid extraction of antioxidants from black bamboo leaves. Food Chemistry. 2014;154:164-70.

10. Camargo J, Rodriguez J. Sitios de estudio y síntesis de los logros obtenidos. Desarrollo tecnológico para optimizar la calidad de los productos obtenidos de bosques de guadua: Definiendo la madurez de los culmos y mejorando los procesos de organización. Pereira, Colombia.2011. p. 27-33.

11. Mosquera O, Rios A, Cortés Y, Niño J. Contenido de azúcares y lignina en culmos de guadua y su estado de madurez. Desarrollo tecnológico para optimizar la calidad de los productos obtenidos de bosques de Guadua: Definiendo la madurez de los culmos y mejorando los procesos de organización. Pereira, Colombia.2011. p. 34-56.

12. Wagner $\mathrm{H}$, Bladt S. Plant drug analysis: $A$ thin layer chromatography atlas. Second edition ed. Germany, Berlín, Verlag: Springer; 1996.

13. Mosquera $O$, Cortés Y, Niño J. Guadua angustifolia en la Ecorregión Cafetera Colombiana. Extracción y cuantificación de lignina insoluble. Recursos Naturales y Ambiente. 2010;61:11-7.

14. Brand-Williams W, Cuvelier ME, Berset C. Use of a free radical method to evaluate antioxidant activity. LWT-Food Science and Technology. 1995;28(1):25-30.

15. Re R, Pellegrini N, Proteggente A, Pannala A, Yang M, Rice-Evans C. Antioxidant activity applying an improved ABTS radical cation decolorization assay. Free Radical Biology and Medicine. 1999;26(9-10):1231-7.

16. Magalhães LM, Santos F, Segundo MA, Reis S, Lima JLFC. Rapid microplate high-throughput methodology for assessment of Folin-Ciocalteu reducing capacity. Talanta. 2010;83(2):441-7.
17. Kim D-O, Jeong SW, Lee CY. Antioxidant capacity of phenolic phytochemicals from various cultivars of plums. Food Chemistry. 2003;81(3):321-6.

18. Turumtay EA, İslamoğlu F, Çavuş $D$, Şahin $H$, Turumtay H, Vanholme B. Correlation between phenolic compounds and antioxidant activity of Anzer tea (Thymus praecox Opiz subsp. caucasicus var. caucasicus). Industrial Crops and Products. 2014;52:687-94.

19. Zhang Y, Wu X, Ren Y, Fu J, Zhang Y. Safety evaluation of a triterpenoid-rich extract from bamboo shavings. Food and Chemical Toxicology. 2004;42(11):1867-75.

20. Zhang Y, Bao B, Lu B, Ren Y, Tie X, Zhang Y. Determination of flavone C-glucosides in antioxidant of bamboo leaves (AOB) fortified foods by reversed-phase high-performance liquid chromatography with ultraviolet diode array detection. Journal of Chromatography $A$. 2005;1065(2):177-85.

21. Augustin JM, Kuzina V, Andersen SB, Bak S. Molecular activities, biosynthesis and evolution of triterpenoid saponins. Phytochemistry. 2011;72(6):435-57.

22. Szakiel A, Pączkowski C, Henry M. Influence of environmental abiotic factors on the content of saponins in plants. Phytochemistry Reviews. 2010;10(4):471-91.

23. Laurichesse $\mathrm{S}$, Avérous L. Chemical modification of lignins: Towards biobased polymers. Progress in Polymer Science. 2014;39(7):1266-90.

24. Kim D-B, Shin G-H, Lee Y-J, Lee JS, Cho J-H, Baik S-O, et al. Assessment and comparison of the antioxidant activities and nitrite scavenging activity of commonly consumed beverages in Korea. Food Chemistry. 2014;151:58-64.

25. Sampietro DA, Catalan C, Vattuone M. Isolation, identification and characterization of allelochemicals/natural products: Science publishers; 2009. 